\title{
Ketamine modulates subgenual cingulate connectivity with the memory-related neural circuit - a mechanism of relevance to resistant depression?
}

Jing J Wong, Owen O'Daly, Mitul A Mehta, Allan H Young, James M Stone

Background: Ketamine has been reported to have efficacy as an antidepressant in several studies of treatment-resistant depression. In this study, we investigate whether an acute administration of ketamine leads to reductions in the functional connectivity of subgenual anterior cingulate cortex (sgACC) with other brain regions. Methods: Thirteen right-handed healthy male subjects underwent a 15 minute resting state fMRI with an infusion of intravenous ketamine (target blood level $=150 \mathrm{ng} / \mathrm{ml}$ ) starting at 5 minutes. We used a seed region centred on the sgACC and assessed functional connectivity before and during ketamine administration. Results: Before ketamine administration, positive coupling with the sgACC seed region was observed in a large cluster encompassing the anterior cingulate and negative coupling was observed with the anterior cerebellum. Following ketamine administration, sgACC activity became negatively correlated with the brainstem, hippocampus, parahippocampal gyrus, retrosplenial cortex, and thalamus. Discussion: Ketamine reduced functional connectivity of the sgACC with brain regions implicated in emotion, memory and mind wandering. It is possible the therapeutic effects of ketamine may be mediated via this mechanism, although further work is required to test this hypothesis. 


\section{Ketamine modulates subgenual cingulate connectivity with the memory-related neural}

2 circuit - a mechanism of relevance to resistant depression?

3 J J Wong (1),

4 Owen G O’Daly (1),

5 Allan H Young (1),

6 Mitul A Mehta (1),

7 James M. Stone (1)*

8 (1) Institute of Psychiatry, Psychology and Neuroscience, King's College London, University of

9 London, London, United Kingdom* Corresponding author

10 email: james.m.stone@kcl.ac.uk

11 Address for correspondence: Centre for Neuroimaging Science, Institute of Psychiatry,

12 Psychology \& Neuroscience, King's College London, De Crespigny Park, London SE5 8AF

13 Telephone: $+44(0) 2075947087$

14 Fax: +44 (0) 2032282477

15 Number of words in abstract: 178

16 Number of words in text: 2540

17 Number of tables: 1

18 Number of figures: 3

19 Number of supplementary materials: 1 
21 Background: Ketamine has been reported to have efficacy as an antidepressant in several

22 studies of treatment-resistant depression. In this study, we investigate whether an acute

23 administration of ketamine leads to reductions in the functional connectivity of subgenual

24 anterior cingulate cortex ( $\mathrm{sgACC})$ with other brain regions.

25 Methods: Thirteen right-handed healthy male subjects underwent a 15 minute resting state

$26 \mathrm{fMRI}$ with an infusion of intravenous ketamine (target blood level $=150 \mathrm{ng} / \mathrm{ml}$ ) starting at 5

27 minutes. We used a seed region centred on the sgACC and assessed functional connectivity

28 before and during ketamine administration.

29 Results: Before ketamine administration, positive coupling with the sgACC seed region was

30 observed in a large cluster encompassing the anterior cingulate and negative coupling was

31 observed with the anterior cerebellum. Following ketamine administration, sgACC activity

32 became negatively correlated with the brainstem, hippocampus, parahippocampal gyrus,

33 retrosplenial cortex, and thalamus.

34 Discussion: Ketamine reduced functional connectivity of the $\mathrm{sgACC}$ with brain regions

35 implicated in emotion, memory and mind wandering. It is possible the therapeutic effects of

36 ketamine may be mediated via this mechanism, although further work is required to test this

37 hypothesis. 


\section{Introduction}

40 The subgenual anterior cingulate (sgACC), or Brodmann area 25 , is a brain region that has been

41 implicated in the control and modulation of mood. Decreased mean sgACC gray matter volume

42 combined with metabolic hyperactivity and hyperconnectivity has been observed in patients with major depressive disorder (MDD) (Davey et al. 2012; Drevets et al. 2008; Greicius et al. 2007;

44 Sundermann et al. 2014). With a growing number of studies demonstrating the association between metabolic hyperactivity in this region and poor therapeutic response (Baeken et al. 2014; Konarski et al. 2009; Maletic \& Raison 2009; Sheline et al. 2010; Taylor \& Liberzon 2007), the activity of this region may prove important for attempts to predict treatment response in patients (Siegle et al. 2012). Furthermore, given that connectivity between the sgACC and the default mode network (particularly the ventromedial prefrontal cortex, and posterior cingulate cortex) has been hypothesised to underlie depressive rumination (Hamilton et al. 2015),

51 therapeutic disruption of this connectivity might also be a potential target for novel

52 antidepressant action.

54 Treatment resistant depression (TRD) is common, with approximately $45 \%$ of patients failing to respond to pharmacological treatment (Papakostas \& Fava 2010). Currently available antidepressants are characterised by a relatively slow onset of effect ranging from weeks to months. There has been great interest in the potential of ketamine as a treatment for TRD patients, given early reports of strong and rapid (within hours) antidepressant properties in 59 patients otherwise resistant to antidepressant treatment (Fond et al. 2014; Salvadore et al. 2009; Zarate et al. 2012; Zarate et al. 2006). Ketamine has been reported to reduce sgACC activity in

61 healthy volunteers (De Simoni et al. 2013; Deakin et al. 2008; Doyle et al. 2013; Stone et al. 
62 2015), and responders to ketamine with treatment resistant bipolar depression have been reported

63 to have increased glucose metabolism in this region (Nugent et al. 2014). However, the effect of

64 ketamine on subgenual connectivity, which might be hypothesised to underlie its antidepressant

65 effects, has not been fully investigated. To date there has only been one study on the effect of

66 ketamine on brain connectivity in relation to potential antidepressant mechanisms. Ketamine was

67 shown to disrupt connectivity between the sgACC and the dorsal nexus 24 hours following

68 administration in healthy volunteers, an effect that was hypothesised to be related to its

69 antidepressant properties (Scheidegger et al. 2012). We hypothesise that immediate effects of

70 ketamine might also play an important part in the therapeutic effects of ketamine in TRD, and

71 that the early modulation of circuits involved in maintenance of depressive cognitions may be

72 necessary for the emergence of objectively measureable clinical improvement.

74 In this study, which is an analysis of existing resting state data (Stone et al. 2015), we

75 investigated the effect of acute intravenous ketamine administration on functional connectivity of 76 the sgACC in healthy volunteers.

\section{Materials and Methods}

79 The study was approved by the East London Research Ethics Committee. Prior to screening for

80 the study, all participants gave written informed consent for inclusion. Inclusion criteria: healthy

81 right-handed male subjects (18-50 years old) with a total body weight $50-100 \mathrm{Kg}$. Exclusion

82 criteria: positive urine drug screen for drugs of abuse, the consumption of more than 5 cups of

83 coffee per day, smoking more than 5 cigarettes per day, taking prescription drugs, and any 
84 history of mental illness, or serious medical condition that, in the opinion of the study doctors,

85 prevented their participation in the study.

86 Thirteen healthy, right handed, male volunteers (age 21-39, mean 27, standard deviation 6.90)

87 were selected for the study after screening. Each subject underwent medical, mental state,

88 physical, and psychiatric examination including electrocardiogram, urine drug screen, and taking

89 measurements of blood pressure, pulse rate, temperature, and weight. Each volunteer underwent

90 venous cannulation in the left antecubital fossa. We attached a $50 \mathrm{ml}$ syringe pump containing

$914 \mathrm{mg} / \mathrm{ml}$ racemic ketamine via an infusion line.

92

93 Image acquisition was conducted, as previously reported (Stone et al. 2015), at the Centre for

94 Neuroimaging Sciences on a General Electric (Milwaukee, Wisconsin) 3-Tesla HDx MRI

95 scanner. Pharmacological MRI (phMRI) blood-oxygen level dependent (BOLD) data were

96 acquired using gradient echo EPI (Echo-Planar Imaging) with parallel imaging accelerated by a

97 factor of 2. Each participant was scanned continuously for 15 minutes to yield a total of 450

98 functional image volumes of 37 , continuous top down, $3 \mathrm{~mm}$ thick slices with a slice gap of 0.3

$99 \mathrm{~mm}$, repeat time (TR) of $2000 \mathrm{~ms}$, echo time (TE) of $30 \mathrm{~ms}$, flip angle of $75^{\circ}$, in-plane resolution

100 of $3.3 \mathrm{~mm}, 64 \times 64$ matrix, and $21.1 \times 21.1 \mathrm{~cm}$ field of view. The ketamine infusion commenced

101 after 5 minutes of resting state acquisition and followed a dynamically modelled intravenous

102 infusion with a target plasma level of $150 \mathrm{ng} / \mathrm{mL}$ determined according to pharmacodynamic

103 properties of ketamine from the "Clements 250 model", with a rapid bolus over 20 seconds of

104 approximately $0.26 \mathrm{mg} / \mathrm{Kg}$, on average, followed by a slow infusion of approximately

$1050.42 \mathrm{mg} / \mathrm{Kg} / \mathrm{Hr}$. Participants' peak ketamine-induced experience was rated by a trained 
106 psychiatrist using the positive and negative syndrome scale (PANSS) immediately following

107 their exit from the scanning room.

108

109 Preprocessing and statistical analyses were performed using Statistical Parametric Mapping

110 software version 8 (SPM8; Wellcome Trust Centre for Neuroimaging, London, England).

111 Functional images were corrected for slice timing effects and subsequently realigned to correct

112 for the effects of volume-to-volume head motion. Data were examined to confirm that framewise

113 translational and rotational head movement did not exceed $2 \mathrm{~mm}$ or 1 degree. Images were co-

114 registered to a high-resolution T1-weighted structural image, and normalised to Montreal

115 Neurological Institute (MNI) space via unified segmentation. The normalised images were

116 smoothed using an 8mm full width at half maximum (FWHM) Gaussian kernel. Additional

117 preprocessing was carried out using the REST toolbox for resting state fMRI analysis (Song et

118 al. 2011). Nuisance variables such as motion parameters (volume to volume translations in 3

119 axes and rotation around these axes), white matter and CSF signal were regressed from the data.

120 The residual time-series was then de-trended and band-pass filtered (frequency range 0.01-

$1210.08 \mathrm{~Hz})$ and a signal time-series was extracted from the sgACC seed $(10 \mathrm{~mm}$ sphere at $[2,28,-5]$

122 based upon a previous publication (Scheidegger et al. 2012)). The 15-minute time series was

123 separated into three 5-minute time-series segments of pre-infusion, early-infusion and late-

124 infusion. The early-infusion portion of the time series was disregarded as any observed

125 connectivity would have been dominated by the phMRI response to the bolus. Finally,

126 connectivity maps between the sgACC seed and the whole brain were created using regression

127 within the REST toolbox, and the resultant r-maps underwent r-to- $Z$ conversion again within

128 REST. 
130 These Z-transformed maps were taken forward into a second level random effect analysis within

131 SPM8. A one sample t-test was used to characterise sgACC connectivity prior to ketamine

132 administration, and a paired t-test was employed (comparing pre- and post-ketamine

133 connectivity) to identify regional changes in sgACC coupling following ketamine administration.

134 We also directly investigated connectivity between the sgACC seed and the dorsal nexus region,

135 by use of two spherical masks of $10 \mathrm{~mm}$ radius at $\mathrm{x}= \pm 36, \mathrm{y}=27, \mathrm{z}=29$ (Sheline et al. 2010).

136 Results were considered significant if they survived family wise error (FWE) correction on the

137 basis of cluster-extent $\left(p_{\mathrm{FWE}}<0.05\right)$. The PANSS general score was tested for normality using the

138 Shapiro-Wilk test prior to regression analysis against ketamine-induced change in sgACC

139 connectivity.

140

\section{Results}

142 Prior to ketamine administration, there was positive coupling $\left(p_{\mathrm{FWE}}<0.05\right)$ between $\operatorname{sgACC}$ and

143 multiple brain regions including anterior cingulate, ventral striatum, and thalamus. There was

144 negative coupling $\left(p_{\mathrm{FWE}}<0.05\right)$ between sgACC and regions including cerebellum, pons,

145 precentral gyrus, superior frontal gyrus, and parahippocampus (Table 1).

146

147 Following ketamine administration, there was significant reduction in sgACC coupling with a

148 large cluster including the hippocampus, retrosplenial cortex (RSC), and thalamus centred at [-2

$149-36]\left(p_{\mathrm{FWE}}=0.002 ; k_{\mathrm{E}}=2885 ; Z_{\mathrm{o}}=3.69\right)$ (Figure 1). Plotting the contrast estimate from the cluster

150 peak, located in the retrosplenial cortex [-6 -553], revealed that the RSC was uncorrelated with

151 the sgACC before ketamine administration, but that it was negatively correlated with the sgACC 
152 following ketamine administration (Figure 2). There was no significant change in connectivity

153 between $\operatorname{sgACC}$ and the dorsal nexus region following ketamine administration.

154

155 Ketamine administration was associated with a mean (SD) increase in PANSS positive, negative, 156 and general subscales to $10.7(2.89), 10.07(3.43)$, and 20.15(3.53) respectively $(\mathrm{p}<0.05)$. Multiple

157 regression analysis using cluster forming threshold of $\mathrm{p}<0.01$ was performed to test for

158 correlations between changes in whole brain functional connectivity with sgACC following

159 ketamine administration and PANSS scores. No correlations with PANSS positive or negative

160 scores were found, but a negative correlation between the PANSS general score and sgACC

161 coupling was observed in the medial prefrontal cortex (mPFC) and subcallosal gyrus (SCG)

$162\left(p_{\mathrm{FWE}}<0.05\right)$. In order to further investigate this correlation, we performed a post-hoc analysis of

163 the correlation between sgACC coupling and depression-related items using the 5 factor PANSS

164 (Lindenmayer et al. 1994). The level of the depression subscale (primarily anxiety and

165 preoccupation rather than the depression item) was significantly increased following ketamine

166 administration $(\mathrm{p}<0.05)$, and was found to be negatively associated with coupling between

167 sgACC and subcallosal gyrus and with right ventrolateral prefrontal cortex $\left(p_{\mathrm{FWE}}<0.05\right)$. The

168 depression subscale was positively associated with coupling between sgACC and right

169 ventromedial prefrontal cortex $\left(p_{\mathrm{FWE}}<0.05\right.$; Figure 3$)$.

170

\section{Discussion}

172 In this study, we examined the acute effect of ketamine on functional connectivity between the

173 sgACC and other brain regions. Our primary aim was to investigate the effect of acute ketamine

174 administration in healthy volunteers on brain networks hypothesised to be involved in the 
175 aetiopathology of depression. The most striking effect of acute ketamine administration in this

176 study is the disruption of connectivity between sgACC and a large cluster encompassing midline

177 thalamus, hippocampus, RSC. This may be of relevance to the antidepressant effect. Both

178 thalamus and hippocampus have been implicated in the pathology of MDD (Malykhin \&

179 Coupland 2015; Yakovlev et al. 1960; Young et al. 2004). Furthermore, the network connectivity

180 between sgACC and the default mode network, including RSC is increased in patients with

181 MDD, and has been suggested to underlie depressive ruminations (Hamilton et al. 2015), a

182 process hypothesized to be of great significance in the maintenance of depressed mood (Disner et

183 al. 2011). If ketamine is able to disrupt the tendency of the mind to return to depressive

184 ruminations through changing the functional connectivity within this network, this may be of

185 particular importance in its antidepressant action. Interestingly, two studies of ketamine effects

186 on functional connectivity revealed an increase rather than a decrease in connectivity between

187 hippocampus and prefrontal cortex following ketamine administration (Gass et al. 2014; Grimm

188 et al. 2015). In addition a graph theory analysis of whole brain connectivity showed a pattern of

189 reduced cortical and increased subcortical/cerebellar connectivity after ketamine in healthy

190 volunteers (Joules et al. 2015). It is worth noting, however, that none of these studies looked at

191 connectivity with sgACC, and it is possible that a decrease in connectivity may have been seen

192 between this region and the hippocampus.

193

194 Although ketamine acutely increased rather than decreased scores on the 5 factor PANSS

195 depression scale in this healthy volunteer sample (it would not have been possible to for

196 depressive scores to reduce as volunteers did not have any symptoms before ketamine

197 administration) it is notable that changes in functional connectivity between subgenual anterior 
198 cingulate and other brain regions correlated only with the general and depressive scores on the

199 PANSS, and not with positive or negative subscales. Our finding of a correlation between

200 ketamine-induced increases on the depression subscale and reduced sgACC coupling with

201 surrounding regions of the SCG is of interest as the SCG is an important node in a neural

202 network comprising of cortical structures, the limbic system, thalamus, hypothalamus, and

203 brainstem nuclei. MDD patients generally show increased activity in SCG, which is normalised

204 by antidepressant treatment (Hamani et al. 2011). It is possible that this local reduction in

205 connectivity reflects a direct consequence of the reduced BOLD activity in this brain region seen

206 following ketamine administration (Stone et al. 2015).

207

208 It is interesting that we found a reduction in connectivity between sgACC and ventrolateral

209 prefrontal cortex associated with increases in ketamine-induced scores on the depression

210 subscale, whereas there was an associated increase in connectivity between sgACC and

211 ventromedial prefrontal cortex connectivity with the same scale. The reason for this is not

212 entirely clear, but both ventrolateral and ventromedial prefrontal cortices have been implicated in

213 depression (Ma et al. 2013; Miller et al. 2015). Ventromedial prefrontal cortex connectivity with

214 sgACC has been suggested to underlie depressive ruminations (Hamilton et al. 2015), so the fact

215 that ketamine acutely increased depression scores and increased this connectivity is in keeping

216 with this finding. In contrast, one study in patients with treatment resistant unipolar depression

217 found increases in connectivity between anterior cingulate cortex and a cluster including

218 ventrolateral prefrontal cortex associated with clinical response to transcranial magnetic

219 stimulation (Baeken et al. 2014), and thus decreases in connectivity between these two regions

220 might be predicted to increase depressive symptoms. However, it is not at all clear how 
221 ketamine-induced increases in depression scores in healthy volunteers may be related to its effect

222 as an antidepressant in patients with existing clinical depression.

223

224 There are a number of limitations regarding the study design. The fact we were studying healthy

225 volunteers means that any effects we see in functional connectivity may not map directly onto

226 those that occur in patients with MDD. Ketamine modulation of brain circuits may vary

227 according to severity of depression and thus networks affected in healthy controls may be

228 different to those suffering from MDD. Related to this, we were not able to measure the

229 antidepressant effect of ketamine as the volunteers had no measureable depressive symptoms at

230 presentation, and so the current data cannot provide evidence that ketamine-induced changes in

231 connectivity are related to ketamine's antidepressant effect. Secondly, the effects investigated in

232 the present study occurred 5 minutes following ketamine administration, during the steady-state

233 infusion, whereas antidepressant effect in patients do not normally arise until 40 minutes to 2

234 hours after ketamine administration. We hypothesise that the changes reported in the current

235 study may be precursors to an antidepressant effect, but it is possible that changes in functional

236 connectivity relevant to antidepressant mechanism between other brain regions might arise at

237 later time points. Thirdly, we did not have a placebo arm. However, participants were not aware

238 when the ketamine was started, and our earlier placebo-controlled study using an identical

239 method of ketamine infusion did not show any effect of placebo on BOLD signal in any brain

240 region (Doyle et al. 2013). Lastly, because the volunteers in this study did not have any

241 depressive symptoms, the correlations of change in connectivity with mood symptoms are

242 difficult to interpret - participants had an increase rather than a decrease in depression-related

243 symptom following ketamine administration. 


\section{Conclusion}

246 We found that ketamine alters the functional connectivity of the sgACC in healthy controls. The

247 networks affected suggest that these changes may be of importance in the therapeutic effects of

248 ketamine in patients with MDD. Further studies in patients are required to test the hypothesis that

249 ketamine may reduce depressive rumination via acute effects on sgACC- RSC connectivity.

250

251

252

253 
255

256

257

258

259

260

261

262

263

264

265

266

267

268

269

270

271

272

273

274

275

276

277

278

279

280

281

282

283

284

285

286

287

288

289

290

291

292

293

294

295

296

297

298

\section{References}

Baeken C, Marinazzo D, Wu GR, Van Schuerbeek P, De Mey J, Marchetti I, Vanderhasselt MA, Remue J, Luypaert R, and De Raedt R. 2014. Accelerated HF-rTMS in treatmentresistant unipolar depression: Insights from subgenual anterior cingulate functional connectivity. World J Biol Psychiatry 15:286-297.

Davey CG, Harrison BJ, Yucel M, and Allen NB. 2012. Regionally specific alterations in functional connectivity of the anterior cingulate cortex in major depressive disorder. Psychol Med 42:2071-2081.

De Simoni S, Schwarz AJ, O'Daly OG, Marquand AF, Brittain C, Gonzales C, Stephenson S, Williams SC, and Mehta MA. 2013. Test-retest reliability of the BOLD pharmacological MRI response to ketamine in healthy volunteers. NeuroImage 64:75-90.

Deakin JF, Lees J, McKie S, Hallak JE, Williams SR, and Dursun SM. 2008. Glutamate and the neural basis of the subjective effects of ketamine: a pharmaco-magnetic resonance imaging study. Arch Gen Psychiatry 65:154-164.

Disner SG, Beevers CG, Haigh EA, and Beck AT. 2011. Neural mechanisms of the cognitive model of depression. Nat Rev Neurosci 12:467-477.

Doyle OM, De Simoni S, Schwarz AJ, Brittain C, O'Daly OG, Williams SC, and Mehta MA. 2013. Quantifying the attenuation of the ketamine pharmacological magnetic resonance imaging response in humans: a validation using antipsychotic and glutamatergic agents. The Journal of pharmacology and experimental therapeutics 345:151-160.

Drevets WC, Savitz J, and Trimble M. 2008. The subgenual anterior cingulate cortex in mood disorders. CNS Spectr 13:663-681.

Fond G, Loundou A, Rabu C, Macgregor A, Lancon C, Brittner M, Micoulaud-Franchi JA, Richieri R, Courtet P, Abbar M, Roger M, Leboyer M, and Boyer L. 2014. Ketamine administration in depressive disorders: a systematic review and meta-analysis. Psychopharmacology (Berl) 231:3663-3676.

Gass N, Schwarz AJ, Sartorius A, Schenker E, Risterucci C, Spedding M, Zheng L, MeyerLindenberg A, and Weber-Fahr W. 2014. Sub-anesthetic ketamine modulates intrinsic BOLD connectivity within the hippocampal-prefrontal circuit in the rat. Neuropsychopharmacology 39:895-906.

Greicius MD, Flores BH, Menon V, Glover GH, Solvason HB, Kenna H, Reiss AL, and Schatzberg AF. 2007. Resting-state functional connectivity in major depression: abnormally increased contributions from subgenual cingulate cortex and thalamus. Biol Psychiatry 62:429-437.

Grimm O, Gass N, Weber-Fahr W, Sartorius A, Schenker E, Spedding M, Risterucci C, Schweiger JI, Bohringer A, Zang Z, Tost H, Schwarz AJ, and Meyer-Lindenberg A. 2015. Acute ketamine challenge increases resting state prefrontal-hippocampal connectivity in both humans and rats. Psychopharmacology (Berl) 232:4231-4241.

Hamani C, Mayberg H, Stone S, Laxton A, Haber S, and Lozano AM. 2011. The subcallosal cingulate gyrus in the context of major depression. Biol Psychiatry 69:301-308.

Hamilton JP, Farmer M, Fogelman P, and Gotlib IH. 2015. Depressive Rumination, the DefaultMode Network, and the Dark Matter of Clinical Neuroscience. Biol Psychiatry.

Joules R, Doyle OM, Schwarz AJ, O'Daly OG, Brammer M, Williams SC, and Mehta MA. 2015. Ketamine induces a robust whole-brain connectivity pattern that can be differentially 
302

303

304

305

306

307

308

309

310

311

312

313

314

315

316

317

318

319

320

321

322

323

324

325

326

327

328

329

330

331

332

333

334

335

336

337

338

339

340

341

342

343

modulated by drugs of different mechanism and clinical profile. Psychopharmacology (Berl) 232:4205-4218.

Konarski JZ, Kennedy SH, Segal ZV, Lau MA, Bieling PJ, McIntyre RS, and Mayberg HS. 2009. Predictors of nonresponse to cognitive behavioural therapy or venlafaxine using glucose metabolism in major depressive disorder. J Psychiatry Neurosci 34:175-180.

Lindenmayer JP, Bernstein-Hyman R, and Grochowski S. 1994. A new five factor model of schizophrenia. Psychiatr Q 65:299-322.

Ma Q, Zeng LL, Shen H, Liu L, and Hu D. 2013. Altered cerebellar-cerebral resting-state functional connectivity reliably identifies major depressive disorder. Brain Res 1495:8694.

Machado-Vieira R, Soares JC, Lara DR, Luckenbaugh DA, Busnello JV, Marca G, Cunha A, Souza DO, Zarate CA, Jr., and Kapczinski F. 2008. A double-blind, randomized, placebo-controlled 4-week study on the efficacy and safety of the purinergic agents allopurinol and dipyridamole adjunctive to lithium in acute bipolar mania. J Clin Psychiatry 69:1237-1245.

Maletic V, and Raison CL. 2009. Neurobiology of depression, fibromyalgia and neuropathic pain. Front Biosci (Landmark Ed) 14:5291-5338.

Malykhin NV, and Coupland NJ. 2015. Hippocampal neuroplasticity in major depressive disorder. Neuroscience.

Miller CH, Hamilton JP, Sacchet MD, and Gotlib IH. 2015. Meta-analysis of Functional Neuroimaging of Major Depressive Disorder in Youth. JAMA psychiatry 72:1045-1053.

Nugent AC, Diazgranados N, Carlson PJ, Ibrahim L, Luckenbaugh DA, Brutsche N, Herscovitch P, Drevets WC, and Zarate CA, Jr. 2014. Neural correlates of rapid antidepressant response to ketamine in bipolar disorder. Bipolar Disord 16:119-128.

Papakostas GI, and Fava M. 2010. Pharmacotherapy for depression and treatment-resistant depression. Hackensack, NJ: World Scientific.

Salvadore G, Cornwell BR, Colon-Rosario V, Coppola R, Grillon C, Zarate CA, Jr., and Manji HK. 2009. Increased anterior cingulate cortical activity in response to fearful faces: a neurophysiological biomarker that predicts rapid antidepressant response to ketamine. Biol Psychiatry 65:289-295.

Scheidegger M, Walter M, Lehmann M, Metzger C, Grimm S, Boeker H, Boesiger P, Henning A, and Seifritz E. 2012. Ketamine decreases resting state functional network connectivity in healthy subjects: implications for antidepressant drug action. PLOS ONE 7:e44799.

Sheline YI, Price JL, Yan Z, and Mintun MA. 2010. Resting-state functional MRI in depression unmasks increased connectivity between networks via the dorsal nexus. Proc Natl Acad Sci U S A 107:11020-11025.

Siegle GJ, Thompson WK, Collier A, Berman SR, Feldmiller J, Thase ME, and Friedman ES. 2012. Toward clinically useful neuroimaging in depression treatment: prognostic utility of subgenual cingulate activity for determining depression outcome in cognitive therapy across studies, scanners, and patient characteristics. Arch Gen Psychiatry 69:913-924.

Song XW, Dong ZY, Long XY, Li SF, Zuo XN, Zhu CZ, He Y, Yan CG, and Zang YF. 2011. REST: a toolkit for resting-state functional magnetic resonance imaging data processing. PLOS ONE 6:e25031.

Stone J, Kotoula V, Dietrich C, De Simoni S, Krystal JH, and Mehta MA. 2015. Perceptual distortions and delusional thinking following ketamine administration are related to 
344

345

346

347

348

349

350

351

352

353

354

355

356

357

358

359

360

361

362

363 increased pharmacological MRI signal changes in the parietal lobe. J Psychopharmacol 29:1025-1028.

Sundermann B, Olde Lutke Beverborg M, and Pfleiderer B. 2014. Toward literature-based feature selection for diagnostic classification: a meta-analysis of resting-state fMRI in depression. Front Hum Neurosci 8:692.

Taylor SF, and Liberzon I. 2007. Neural correlates of emotion regulation in psychopathology. Trends Cogn Sci 11:413-418.

Yakovlev P, Locke S, Koskoff D, and Patton R. 1960. Limbic nuclei of thalamus and connections of limbic cortex. Archives of neurology 3:620-641.

Young KA, Holcomb LA, Yazdani U, Hicks PB, and German DC. 2004. Elevated neuron number in the limbic thalamus in major depression. Am J Psychiatry 161:1270-1277.

Zarate CA, Jr., Brutsche NE, Ibrahim L, Franco-Chaves J, Diazgranados N, Cravchik A, Selter J, Marquardt CA, Liberty V, and Luckenbaugh DA. 2012. Replication of ketamine's antidepressant efficacy in bipolar depression: a randomized controlled add-on trial. Biol Psychiatry 71:939-946.

Zarate CA, Jr., Singh JB, Carlson PJ, Brutsche NE, Ameli R, Luckenbaugh DA, Charney DS, and Manji HK. 2006. A randomized trial of an N-methyl-D-aspartate antagonist in treatment-resistant major depression. Arch Gen Psychiatry 63:856-864. 


\section{Table $\mathbf{1}$ (on next page)}

Resting state sgACC coupling prior to ketamine administration

Regions showing significant coupling with sgACC prior to ketamine infusion ( $p F W E<0.05$ corrected for multiple comparisons on the basis of cluster extent, using a cluster-forming threshold of $z=3 \cdot 1$ ). 


\begin{tabular}{|c|c|c|c|c|c|c|c|c|}
\hline \multirow{2}{*}{ sgACC Coupling } & \multirow{2}{*}{ Brain Region } & \multirow{2}{*}{ Brodmann Area } & \multicolumn{2}{|c|}{ Cluster-level } & \multirow{2}{*}{$\begin{array}{c}\text { Peak-level } \\
\left(Z_{\underline{o}}\right)\end{array}$} & \multicolumn{3}{|c|}{ Coordinates } \\
\hline & & & $p_{\mathrm{FWE}}$ & $k_{\mathrm{E}}$ & & $\mathrm{x}$ & $\mathrm{y}$ & $\mathrm{z}$ \\
\hline Positive & Ventral Anterior Cingulate & 24 & $<0.001$ & 12610 & 6.69 & 4 & 27 & -6 \\
\hline Positive & Dorsal Anterior Cingulate & 32 & & & 6.23 & -6 & 35 & -3 \\
\hline Positive & Thalamus & & & & 5.79 & -2 & -3 & -3 \\
\hline Negative & Anterior Cerebellum & & $<0.001$ & 3705 & 4.69 & -12 & -37 & -27 \\
\hline Negative & Pons & & & & 4.16 & 9 & -22 & -24 \\
\hline Negative & Anterior Cerebellum & & & & 3.96 & 10 & -37 & -26 \\
\hline Negative & Middle Frontal Gyrus & 9 & $<0.001$ & 1089 & 4.68 & 43 & 12 & 33 \\
\hline Negative & Middle Frontal Gyrus & 9 & & & 4.50 & 42 & 3 & 39 \\
\hline Negative & Precentral Gyrus & 6 & & & 4.03 & 51 & 0 & 40 \\
\hline Negative & Superior Frontal Gyrus & 10 & 0.001 & 971 & 4.45 & 22 & 51 & 24 \\
\hline Negative & Middle Frontal Gyrus & 9 & & & 4.12 & 34 & 29 & 28 \\
\hline Negative & Middle Frontal Gyrus & 9 & & & 3.77 & 30 & 36 & 21 \\
\hline Negative & Inferior Parietal Lobule & 40 & 0.007 & 649 & 4.17 & 66 & -46 & 31 \\
\hline Negative & Inferior Parietal Lobule & 40 & & & 3.46 & 70 & -46 & 22 \\
\hline Negative & Postcentral Gyrus & 2 & & & 3.44 & 61 & -30 & 43 \\
\hline
\end{tabular}

1 
1

sgACC connectivity following ketamine administration.

Regions showing significant ( $p F W E<0.05$ ) reduction in sgACC coupling following ketamine administration (red/yellow).

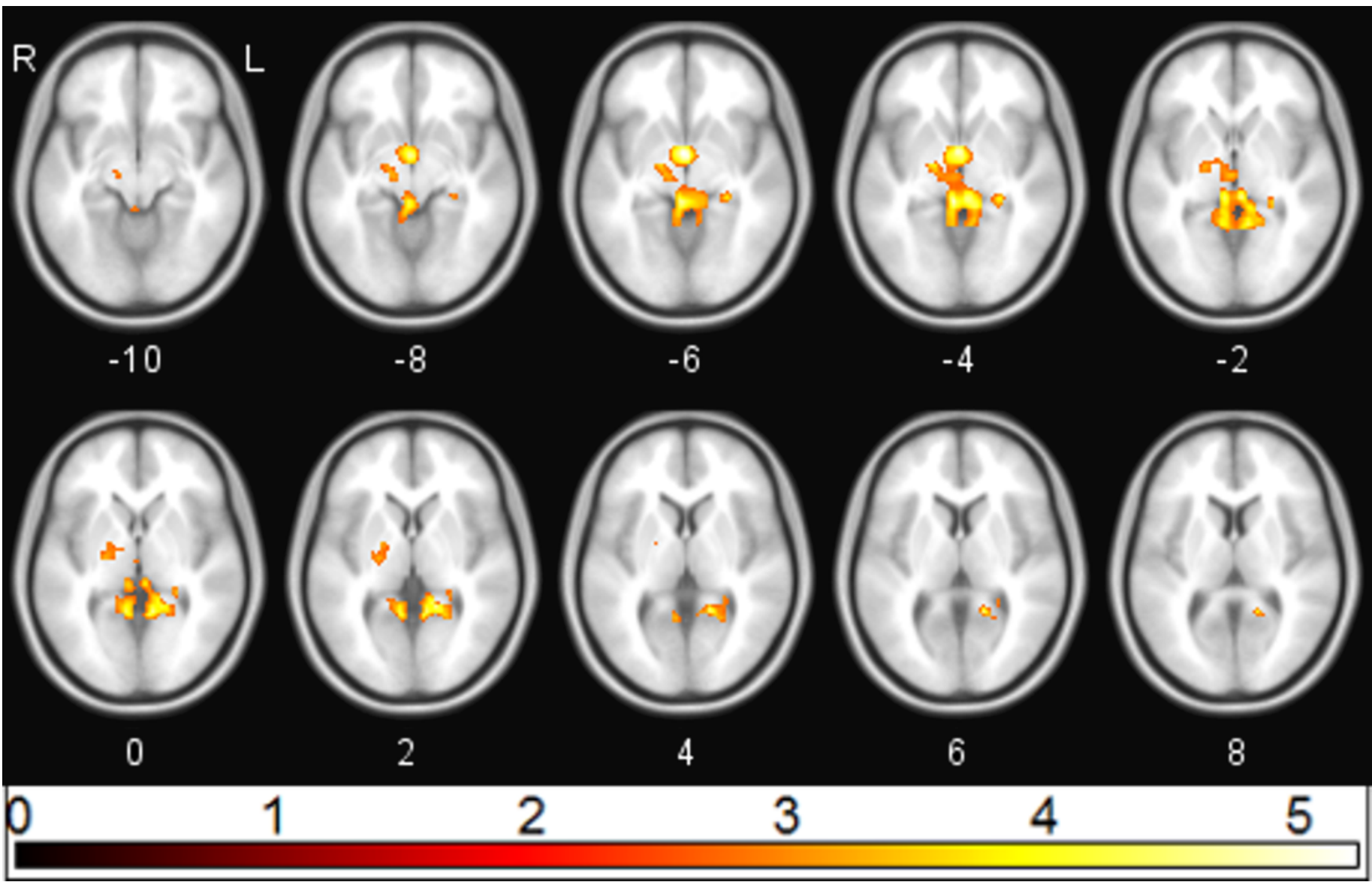


2

Effect of ketamine on connectivity between SgACC and retrosplenial cortex

Correlation between SgACC and retrosplenial cortex [ -6 - -553 3 before and after the start of ketamine administration.

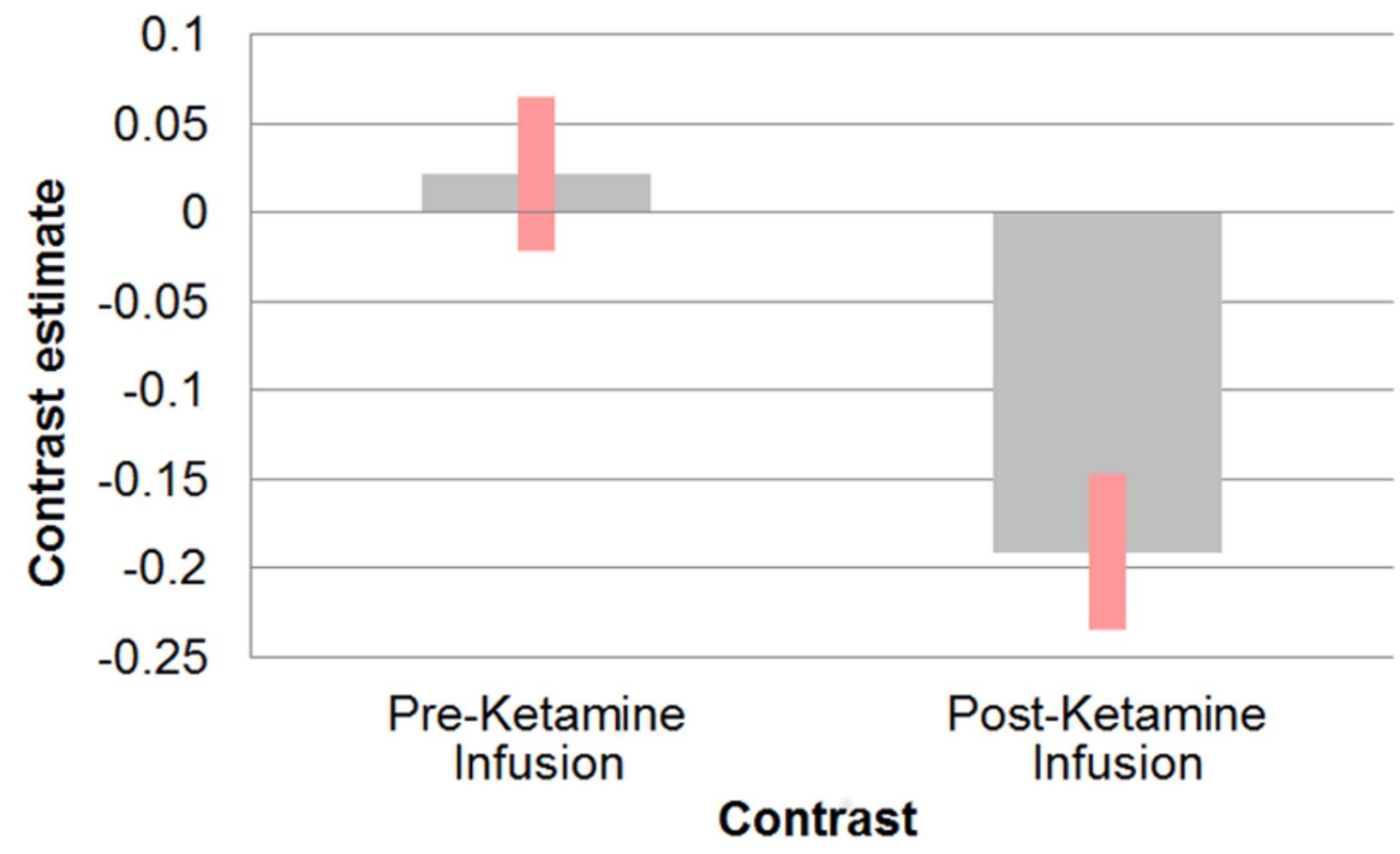




\section{3}

Correlation between PANSS depression and sgACC coupling

Regions showing significant ( $p F W E<0.05$ ) correlations between PANSS depression score and change in sgACC coupling following ketamine administration (blue - negative correlation, yellow - positive correlation).

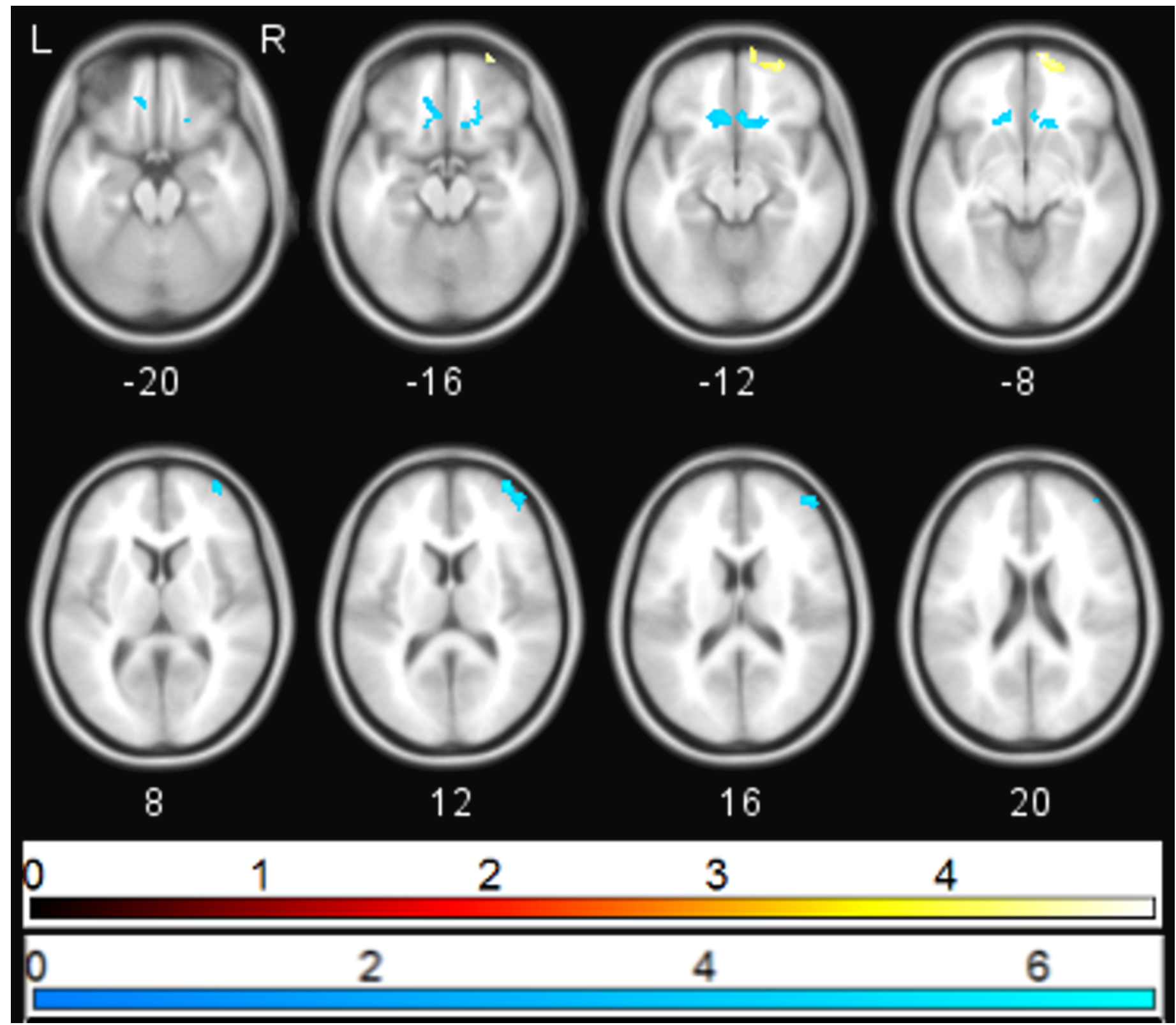

